\title{
AMPLENESS OF CANONICAL DIVISORS OF HYPERBOLIC NORMAL PROJECTIVE VARIETIES
}

\author{
FEI HU, SHENG MENG, AND DE-QI ZHANG
}

\begin{abstract}
Let $X$ be a projective variety which is algebraic Lang hyperbolic. We show that Lang's conjecture holds (one direction only): $X$ and all its subvarieties are of general type and the canonical divisor $K_{X}$ is ample at smooth points and Kawamata log terminal points of $X$, provided that $K_{X}$ is $\mathbb{Q}$-Cartier, no Calabi-Yau variety is algebraic Lang hyperbolic and a weak abundance conjecture holds.
\end{abstract}

\section{INTRODUCTION}

We work over the field $\mathbb{C}$ of complex numbers. A variety $X$ is Brody hyperbolic (resp. algebraic Lang hyperbolic) if every holomorphic map $V \rightarrow X$, where $V$ is the complex line $\mathbb{C}$ (resp. $V$ is an abelian variety), is a constant map. Since an abelian variety is a complex torus, Brody hyperbolicity implies algebraic Lang hyperbolicity. When $X$ is a compact complex variety, Brody hyperbolicity is equivalent to the usual Kobayashi hyperbolicity (cf. [13]).

In the first part (Theorem 1.4 and its consequences 3.7, 3.8) of this paper, we let $X$ be a normal projective variety and aim to show the ampleness of the canonical divisor $K_{X}$ of $X$, assuming that $X$ is algebraic Lang hyperbolic. We allow $X$ to have arbitrary singularities and assume only that $X$ is $\mathbb{Q}$-Gorenstein (so that the ampleness of $K_{X}$ is well-defined), i.e., $K_{X}$ is $\mathbb{Q}$-Cartier: $m K_{X}$ is a Cartier divisor for some positive integer $m$.

For related work, it was proven in [17] that a 3-dimensional hyperbolic smooth projective variety $X$ has ample $K_{X}$ unless $X$ is a Calabi-Yau manifold where every non-zero effective divisor is ample. The authors of [7] proved the ampleness of $K_{X}$ when $X$ is a smooth projective threefold having a Kähler metric of negative holomorphic sectional curvature; they also generalized the results to higher dimensions with some additional conditions.

In the second part of the paper (Theorem 1.5 and its more general form Theorem 3.1), we make some contributions toward Lang's conjecture in Corollary 1.6, where even the

2000 Mathematics Subject Classification. 32Q45, 14E30.

Key words and phrases. algebraic Lang hyperbolic variety, ample canonical divisor. 
normality of $X$ is not assumed. Our approach is to take a projective resolution of $X$ and run the relative Minimal Model Program (MMP) over $X$. We use only the frame work of MMP, but not its detailed technical part. Certain mild singularities occur naturally along the way. See [12, Definitions 2.34 and 2.37] for definitions of canonical, Kawamata log terminal (klt), and divisorial log terminal (dlt) singularities.

In the last part (Proposition 1.7 and its more general form Theorem [3.2), we try to avoid assuming conjectures.

We now state two conjectures. Conjecture 1.1 below is long standing. When $\operatorname{dim} X \leq 2$, it is true by the classification of complex surfaces and the following:

Fact $(*)$. A (smooth) K3 surface has infinitely many (singular) elliptic curves; see [15, Theorem in Appendix] or Proposition 2.2.

In Conjecture 1.1, the conclusion means the existence of at least one non-constant holomorphic map $f: V \rightarrow X$ from an abelian variety $V$, but does not require the image $f(V)$ (or the union of such images) to be Zariski-dense in $X$. This does not seem sufficient for our purpose to show the non-existence of subvariety $X^{\prime}$ of Kodaira dimension zero in an algebraic Lang hyperbolic variety $W$ as in Corollary 1.6 below (see 1.9, and think about a proof of the non-hyperbolicity of every normal $K 3$ surface using the Fact $(*)$ above). Fortunately, we are able to show in Corollary 1.6 (or Theorems 1.5 and 3.1) that the normalization $X$ of $X^{\prime} \subseteq W$ is a Calabi-Yau variety and hence $f$ composed with the finite morphism $X \rightarrow X^{\prime} \subseteq W$ produces a non-constant holomorphic map from the abelian variety $V$, thus deducing a contradiction to the hyperbolicity of $W$.

Conjecture 1.1. Let $X$ be an absolutely minimal Calabi-Yau variety (cf. 2.1). Suppose further that every birational morphism $X \rightarrow Y$ onto a normal projective variety is an isomorphism. Then $X$ is not algebraic Lang hyperbolic.

We need the result below about nef reduction map and nef dimension. A meromorphic map $f: X \rightarrow Y$ between complex varieties is almost holomorphic if it is well defined on a Zariski dense open subset $U$ of $X$ and the map $f_{\mid U}: U \rightarrow Y$ has compact connected general fibres.

Theorem 1.2. (cf. [1, Theorem 2.1]) Let $L$ be a nef $\mathbb{Q}$-Cartier divisor on a normal projective variety $X$. Then there exists an almost holomorphic, dominant rational map $f: X \rightarrow Y$ with connected fibres, called a "nef reduction map" such that

(1) $L$ is numerically trivial on all compact fibres $F$ of $f$ with $\operatorname{dim} F=\operatorname{dim} X-\operatorname{dim} Y$;

(2) for every general point $x \in X$ and every irreducible curve $C$ passing through $x$ with $\operatorname{dim} f(C)>0$, we have L.C $>0$. 
The map $f$ is unique up to birational equivalence of $Y$. We call $\operatorname{dim} Y$ the "nef dimension" of $L$ and denote it as $n(L)$.

Proof. See [1] for the proof.

Next we state Conjecture 1.3. We stress that 1.3 without the extra "Hyp(A)" is the usual abundance conjecture and stronger than our one here. When $K_{X}$ is nef and big or when $\operatorname{dim} X \leq 3$, both Conjectures 1.3 (1) and 1.3 (2) (and their log version, even without the extra Hyp(A)) are true; see [12, Theorem 3.3, §3.13], or Proposition 2.2.

Conjecture 1.3. Let $X$ be an n-dimensional minimal normal projective variety, i.e., the canonical divisor $K_{X}$ is a nef $\mathbb{Q}$-Cartier divisor. Assume Hyp $(A)$ : the nef dimension $n\left(K_{X}\right)$ satisfies $n\left(K_{X}\right)=n$.

(1) If $X$ has at worst klt singularities, then $K_{X}$ is semi-ample, i.e., the linear system $\left|m K_{X}\right|$ is base-point free for some $m>0$.

(2) If $X$ has at worst canonical singularities and $K_{X} \not \equiv 0$ (not numerically zero), then the Kodaira dimension $\kappa(X)>0$.

Theorems 1.4 and 1.5 below are our main results. When $X$ has at worst klt singularities, Theorem 1.4 below follows from the MMP and has been generalized to the quasi-projective case in [14. In Theorem 1.4, we do not impose any condition on the singularities of $X$, except the $\mathbb{Q}$-Cartierness of $K_{X}$. This assumption is necessary to formulate the conclusion that $K_{X}$ be ample. Without assuming Conjecture 1.1 or 1.3 as in Theorem 1.4, we can at least say that $K_{X}$ is movable or nef in codimension-one (cf. Remark 1.8). See also Corollaries 3.7 and 3.8 when $\operatorname{dim} X \leq 3$.

Theorem 1.4. Let $X$ be a $\mathbb{Q}$-Gorenstein normal projective variety which is algebraic Lang hyperbolic. Assume that Conjecture 1.1 holds for all varieties birational to $X$, or to any subvariety of $X$. Further, assume that Conjecture 1.3(1) holds for all varieties birational to $X$.

Then $K_{X}$ is ample at smooth points and klt points of $X$. To be precise, there is a birational morphism $f_{c}: X_{c} \rightarrow X$ such that $X_{c}$ has at worst klt singularities, $K_{X_{c}}$ is ample, and $E_{c}:=f_{c}^{*} K_{X}-K_{X_{c}}$ is an effective and $f_{c}$-exceptional divisor with $f_{c}\left(E_{c}\right) \subseteq$ $\operatorname{Nklt}(X)$, the non-klt locus of $X$.

In particular, $f_{c}=\mathrm{id}$ and $K_{X}$ is ample, if $X$ has at worst klt singularities.

The normality of $X$ is not assumed in Theorem 1.5 below which is a special case of Theorem 3.1 by letting $g: X \rightarrow W$ there to be the identity map id $X: X \rightarrow X$. When $\operatorname{dim} X \leq 3$, Case (3) below does not occur. 
Theorem 1.5. Let $X$ be an algebraic Lang hyperbolic projective variety of dimension $n$. Assume either $n \leq 3$ or Conjecture 1.3](2) (resp. either $n \leq 3$ or Conjecture 1.3(2) without the extra $\operatorname{Hyp}(A))$ holds for all varieties birational to $X$.

Then there is a birational surjective morphism $g_{m}: X_{m} \rightarrow X$ such that $X_{m}$ is a minimal variety with at worst canonical singularities and one of the following is true.

(1) $K_{X_{m}}$ is ample. Hence both $X_{m}$ and $X$ are of general type.

(2) $g_{m}: X_{m} \rightarrow X$ is the normalization map. $X_{m}$ is an absolutely minimal Calabi-Yau variety with $\operatorname{dim} X_{m} \geq 3$.

(3) There is an almost holomorphic map $\tau: X_{m} \rightarrow Y$ (resp. a holomorphic map $\tau: X_{m} \rightarrow Y$ ) such that its general fibre $F$ is an absolutely minimal CalabiYau variety with $3 \leq \operatorname{dim} F<\operatorname{dim} X_{m}$, and $\left(g_{m}\right)_{\mid F}: F \rightarrow g_{m}(F) \subset X$ is the normalization map.

Given a projective variety $W$, let $\widetilde{W} \stackrel{\sigma}{\rightarrow} W$ be a projective resolution. We define the albanese variety of $W$ as $\operatorname{Alb}(W):=\operatorname{Alb}(\widetilde{W})$, which is independent of the choice of the resolution $\widetilde{W}$, since every two resolutions of $W$ are dominated by a third one and the albanese variety, being an abelian variety, contains no rational curves. We define the albanese (rational) map alb $_{W}: W \rightarrow \operatorname{Alb}(W)$ as the composition

$$
W_{-\rightarrow}^{\sigma^{-1}} \widetilde{W} \stackrel{\mathrm{alb}_{\widetilde{W}}}{\longrightarrow} \operatorname{Alb}(\widetilde{W}) \text {. }
$$

One direction of Lang's [13, Conjecture 5.6] follows from Conjectures 1.1] and 1.3 (2). See Remark 1.8 (6) for the other direction.

Corollary 1.6. Let $W$ be an algebraic Lang hyperbolic projective variety of dimension $n$. Assume either $n \leq 3$ or Conjecture 1.3(2) holds for all varieties of dimension $\leq n$. Then we have:

(1) If Conjecture 1.1 holds for all varieties of dimension $\leq n$, then $W$ and all its subvarieties are of general type.

(2) If the albanese map $\mathrm{alb}_{W}: W \rightarrow \mathrm{Alb}(W)$ has general fibres of dimension $\leq 2$, then $W$ is of general type.

Without assuming Conjecture 1.3 (or 1.1), we have the following (see Theorem 3.2 for a generalization). For a singular projective variety $Z$, we define the Kodaira dimension $\kappa(Z)$ as $\kappa(\widetilde{Z})$ (cf. [12, §7.73]) for some (or equivalently any) projective resolution $\widetilde{Z} \rightarrow Z$.

Proposition 1.7. Let $X$ be an algebraic Lang hyperbolic projective variety. Assume one of the following conditions.

(i) $X$ has maximal albanese dimension, i.e., the albanese map $\operatorname{alb}_{X}: X \rightarrow \operatorname{Alb}(X)$ is generically finite (but not necessarily surjective). 
(ii) The Kodaira dimension $\kappa(X) \geq \operatorname{dim} X-2$.

(iii) $\kappa(X) \geq \operatorname{dim} X-3$, and Conjecture 1.1 holds in dimension three.

Then $X$ is of general type.

Remark 1.8. (1) In Theorem 1.4, by the equality $f_{c}^{*} K_{X}=K_{X_{c}}+E_{c}$ and the ampleness of $K_{X_{c}}$, the exceptional locus $\operatorname{Exc}\left(f_{c}\right)$ (the subset of $X_{c}$ along which $f_{c}$ is not isomorphic) is contained in Supp $E_{c}$. Indeed, if $C$ is an $f_{c}$-contractible curve, then $0=C \cdot f_{c}^{*} K_{X}=$ $C . K_{X_{c}}+C . E_{c}>C . E_{c}$, so $C \subseteq \operatorname{Supp} E_{c}$. This and the effectivity of $E_{c}$ justifies the phrasal: $K_{X}$ is ample outside $f_{c}\left(E_{c}\right)$.

(2) Without assuming Conjecture 1.1 or 1.3, the proof of Theorem 1.4 (Claim 3.3 and the equality (2) above it) shows that $\left(f^{\prime}\right)^{*} K_{X}=K_{X^{\prime}}+E^{\prime}$ with $K_{X^{\prime}}$ nef and $E^{\prime} \geq 0$ $f^{\prime}$-exceptional. Hence $K_{X}=f_{*}^{\prime} K_{X^{\prime}}$ is movable, or nef in codimension-one.

(3) Let $X_{2} \rightarrow X_{1}$ be a finite morphism (but not necessarily surjective). If $X_{1}$ is Brody hyperbolic or algebraic Lang hyperbolic then so is $X_{2}$. The converse is not true.

(4) Every algebraic Lang hyperbolic projective variety $X_{1}$ is absolutely minimal in the sense of 2.1, i.e., every birational map $h: X_{2} \rightarrow X_{1}$ from a normal projective variety $X_{2}$ with at worst klt singularities, is a well defined morphism. This result was proved by S. Kobayashi when $X_{2}$ is nonsingular. Indeed, let $Z$ be a resolution of the graph of $h$ such that we have a birational surjective morphisms $p_{i}: Z \rightarrow X_{i}$ satisfying $h \circ p_{2}=p_{1}$. Then every fibre $p_{2}^{-1}\left(x_{2}\right)$ is rationally chain connected by [6, Corollary 1.5] and hence $p_{1}\left(p_{2}^{-1}\left(x_{2}\right)\right)$ is a point since hyperbolic $X_{1}$ contains no rational curve. Thus $h$ can be extended to a well defined morphism by [8, Proof of Lemma 14], noting that $X_{2}$ is normal and $p_{2}$ is surjective, and using the Stein factorization.

(5) If $Y$ is an algebraic Lang hyperbolic Calabi-Yau variety (like $X_{m}$ and $F$ in Theorem 1.5 (2) and (3), respectively), then every birational morphism $h: Y \rightarrow Z$ onto a normal projective variety is an isomorphism. Indeed, by [11, Corollary 1.5], $Z$ has only canonical singularities. Thus the exceptional locus $\operatorname{Exc}(h)$ is covered by rational curves by $[6$, Corollary 1.5]. Since $Y$ is hyperbolic and hence has no rational curve, we have $\operatorname{Exc}(h)=\emptyset$ and hence $h$ is an isomorphism, $Z$ being normal and by the Stein factorization.

(6) Consider the converse of Corollary [1.6, i.e., the other direction of Lang [13, Conjecture 5.6], but with the assumption that every non-uniruled projective variety has a minimal model with at worst canonical singularities and that abundance Conjecture 1.3 (2) holds. To be precise, supposing that a projective variety $W$ and all its subvarieties are of general type, we see that $W$ is algebraic Lang hyperbolic. Indeed, let $f: V \rightarrow W$ be a morphism from an abelian variety $V$ and let $V \rightarrow X \rightarrow f(V)$ be its Stein factorization, where $V \rightarrow X$ has a connected general fibre $F$ and $X \rightarrow f(V)$ is a finite morphism. Since $V$ is non-uniruled, so is $F$. Hence $\kappa(F) \geq 0$ by the assumption. The assumption and 
Iitaka's $C_{n, m}$ also imply that $0=\kappa(V) \geq \kappa(F)+\kappa(X) \geq \kappa(X) \geq \kappa(f(V))=\operatorname{dim} f(V)$ (cf. [9, Corollary 1.2]). Hence $f$ is a constant map.

1.9. Comments about the proofs. In our proofs, neither the existence of minimal model nor the termination of MMP is assumed. Let $W$ be an algebraic Lang hyperbolic projective variety. To show that every subvariety $X$ of $W$ is of general type, one key observation is the existence of a birational model $X^{\prime}$ of $X$ with $K_{X^{\prime}}$ relative nef over $W$, by using the main Theorem 1.2 in [2]. $K_{X^{\prime}}$ is indeed nef since $W$ is hyperbolic (cf. Lemma 2.5 or 2.6). One natural approach is to take a general fibre $F$ (which may not even be normal) of an Iitaka (rational) fibration of $X$ (assuming $\kappa(X) \geq 0$ ) and prove that $F$ has a minimal model $F_{m}$. Next, one tries to show that $q\left(F_{m}\right)=0$ and $F_{m}$ is a Calabi-Yau variety and then tries to use Conjecture 1.1 to produce a non-hyperbolic subvariety $S$ of $F_{m}$, but this does not guarantee the same on $F \subset X$ (to contradict the hyperbolicity of $X$ ) because such $S \subseteq F_{m}$ might be contracted to a point on $F$. In our approach, we are able to show that the normalization of $F$ is a Calabi-Yau variety, which is the key of the proofs. It would not help even if one assumes the smoothness of the ambient space $W$ since its subvariety $X$ may not be smooth, or at least normal or Cohen-Macaulay to define the canonical divisor $K_{X}$ meaningfully to pull back or push forward.

Acknowledgement. We would like to thank the referee for very careful reading, many suggestions to improve the paper and the insistence on clarity of exposition. The last named-author is partially supported by an ARF of NUS.

\section{Preliminary Results}

\subsection{Convention, notation and terminology}

In this paper, by hyperbolic we mean algebraic Lang hyperbolic.

(i) We use the notation and terminology in the book of Hartshorne and the book [12].

(ii) Given two morphisms $g_{i}: Y_{i} \rightarrow Z(i=1,2)$ between varieties, a rational map $Y_{1} \rightarrow Y_{2}$ is said to be a map over $Z$, if the composition $Y_{1} \rightarrow Y_{2} \stackrel{g_{2}}{\rightarrow} Z$ coincides with $g_{1}: Y_{1} \rightarrow Z$.

(iii) For a rational map $h: X \rightarrow Y$, we take a birational resolution $\pi: W \rightarrow X$ of the indeterminacy of $h$ such that the composition $h \circ \pi$ is a well defined morphism: $h_{1}: W \rightarrow Y$. For a point $y \in Y$, we defined the fibre $h^{-1}(y)$ as $\pi\left(h_{1}^{-1}(y)\right)$. This definition does not depend on the choice of the resolution $\pi$ of $h$, since every two such resolutions are dominated by a third one. 
(iv) For a singular projective variety $Z$, we define the Kodaira dimension $\kappa(Z)$ as $\kappa(\widetilde{Z})$ (cf. [12, §7.73]) for some (or equivalently any) projective resolution $\widetilde{Z} \rightarrow Z$. When $\kappa(\widetilde{Z}) \geq 0$, there is a (rational) Iitaka fibration, unique up to birational equivalence, $I_{Z}: Z \rightarrow Y$ such that its very general fibre $F$ has $\kappa(F)=0$ and that $\operatorname{dim} Y=\kappa(Z)$.

(v) For two Weil $\mathbb{Q}$-divisors $D_{i}$ on a normal variety $X$, if $m\left(D_{1}-D_{2}\right) \sim 0$ (linear equivalence) for some integer $m>0$, we say that $D_{1}$ and $D_{2}$ are $\mathbb{Q}$-linearly equivalent and denote this relation as: $D_{1} \sim_{\mathbb{Q}} D_{2}$.

(vi) Let $X$ be a normal projective variety. $X$ is a Calabi-Yau variety if $X$ has at worst canonical singularities, its canonical divisor is $\mathbb{Q}$-linearly equivalent to zero: $K_{X} \sim_{\mathbb{Q}} 0$, and the irregularity $q(X):=h^{1}\left(X, \mathcal{O}_{X}\right)=0$. If this is the case, $X$ has Kodaira dimension $\kappa(X)=0$.

(vii) A projective variety $X$ is of general type if some (equivalently every) projective resolution $X^{\prime}$ of $X$ has maximal Kodaira dimension: $\kappa\left(X^{\prime}\right)=\operatorname{dim} X^{\prime}$.

(viii) A $\mathbb{Q}$-Gorenstein variety $X$ is minimal if the canonical divisor $K_{X}$ is numerically effective $(=n e f)$. A projective variety $X_{1}$ is absolutely minimal if every birational map $h: X_{2} \rightarrow X_{1}$ from a normal projective variety $X_{2}$ with at worst klt singularities, is a well defined morphism.

Proposition 2.2. (1) Let $X$ be a projective surface. Then either it has infinitely many rational curves or elliptic curves, or it is of general type, or it is birational to a simple abelian surface.

(2) Let $Y$ be a normal projective surface such that $K_{Y} \sim_{\mathbb{Q}} 0$ and $Y$ is birational to an abelian surface $A$. Then $Y$ is isomorphic to $A$.

(3) Let $Z$ be a normal projective surface with $K_{Z} \sim_{\mathbb{Q}} 0$. Then $Z$ is not algebraic Lang hyperbolic. In particular, Conjecture 1.1 holds when dimension $\leq 2$.

(4) In dimension $\leq 3$, both Conjectures 1.3 (1) and (2) even without the extra Hyp (A) (and even for log canonical pairs) hold.

(5) Both Conjectures 1.3 (1) and (2) even without the extra Hyp(A) hold for varieties of general type.

(6) Let $X$ be a variety with maximal albanese dimension, i.e., $\operatorname{dim} \operatorname{alb}_{X}(X)=\operatorname{dim} X$. If $X$ has only canonical singularities and $K_{X}$ is nef, then $K_{X}$ is semi-ample. In particular, Conjecture 1.3 (2) even without the extra Hyp(A) holds for varieties with maximal albanese dimension.

Proof. (1) It is well known that every Enrique surface has an elliptic fibration. By [15, Theorem in Appendix], every $K 3$ surface has infinitely many singular elliptic curves. Thus (1) follows from the classification of algebraic surfaces. 
(2) Take a common resolution $Z$ of $Y$ and $A$, i.e., let $p: Z \rightarrow A$ and $q: Z \rightarrow Y$ be two biraitonal morphisms. Write $K_{Z}=p^{*} K_{A}+E_{p}=E_{p}$ where $E_{p} \geq 0$ is $p$-exceptional and $\operatorname{Supp} E_{p}$ is equal to $\operatorname{Exc}(p)$, the exceptional locus of $p$. Write $K_{Z}=q^{*} K_{Y}+E_{1}-$ $E_{2} \sim_{\mathbb{Q}} E_{1}-E_{2}$ where both $E_{i} \geq 0$ are $q$-exceptional and there is no common irreducible component of $E_{1}$ and $E_{2}$.

Equating the two expressions of $K_{Z}$, we get $E_{1} \sim_{\mathbb{Q}} E_{2}+E_{p}$. Since $E_{1}$ is $q$-exceptional, its Iitaka $D$-dimension is zero, so $E_{1}=E_{2}+E_{p}$. Thus $\operatorname{Exc}(p)=\operatorname{Supp} E_{p} \subseteq \operatorname{Supp} E_{1} \subseteq$ $\operatorname{Exc}(q)$. Hence there is a birational surjective morphism $h: A \rightarrow Y$ such that $q=h \circ p$ :

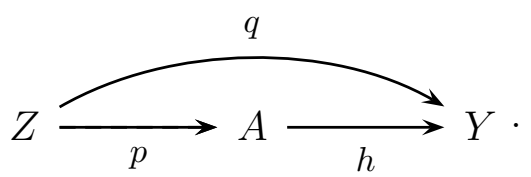

If $h: A \rightarrow Y$ is not an isomorphism, then it contracts a curve $C$ on $A$ to a point on $Y$. Clearly, $C^{2}<0$. By the genus formula, $2 g(C)-2=C^{2}+C . K_{A}=C^{2}<0$. So $C \cong \mathbb{P}^{1}$. This contradicts the fact that there is no rational curve on the abelian variety $A$. Thus $h$ is an isomorphism.

(3) Since $K_{Z} \sim_{\mathbb{Q}} 0, Z$ is not of general type. By (1), either $Z$ is birational to an abelian surface, or $Z$ has infinitely many rational or elliptic curves. In the first case, $Z$ is an abelian surface by (2). Thus $Z$ is not algebraic Lang hyperbolic in all cases.

(4) We refer to [12, §3.13] for its proof or references.

(5) This follows from the base point freeness result for nef and big canonical divisors of klt varieties (cf. [12, Theorem 3.3]).

(6) It is proven in [5, Theorem 3.6].

The result below is just [9, Theorem 8.3]; see also [9, Lemma 8.1] and [8, Theorem 1] for the assertion (1).

Lemma 2.3. Let $X$ be a normal projective variety with only canonical singularities and $K_{X} \sim_{\mathbb{Q}} 0$. Suppose that the irregularity $q(X)>0$. Then we have:

(1) The albanese map $\operatorname{alb}_{X}: X \rightarrow A:=\operatorname{Alb}(X)$ is a surjective morphism, where $\operatorname{dim} A=q(X)$.

(2) There is an étale morphism $B \rightarrow A$ from another abelian variety $B$ such that the fibre product $X \times_{A} B \cong Z \times B$ for some variety $Z$.

(3) $X$ is covered by images of abelian varieties $\{z\} \times B(z \in Z)$.

Lemma 2.4. Let $X$ be a normal projective variety of dimension $n$ such that $K_{X}$ is $\mathbb{Q}$ Cartier. Suppose that $X$ is not uniruled and $K_{X} \equiv 0$ (numerically). Then $X$ has at worst canonical singularities and $K_{X} \sim_{\mathbb{Q}} 0$. 
Proof. Let $\gamma: \widetilde{X} \rightarrow X$ be a projective resolution and write $K_{\widetilde{X}}=\gamma^{*} K_{X}+\left(E_{1}-E_{2}\right) \equiv$ $E_{1}-E_{2}$ such that $E_{i} \geq 0(i=1,2)$ are $\gamma$-exceptional and have no common components. Since $X$ and hence $\widetilde{X}$ are non-uniruled, $K_{\widetilde{X}}$ is pseudo-effective by [3, Theorem 2.6]. Let $K_{\tilde{X}}=P_{1}+N_{1}$ be the $\sigma$-decomposition in [16, ChIII, $\left.\S 1 . \mathrm{b}\right]$, which is also called the Zariski decomposition in codimension-one. Here $P_{1}$ is the movable part and $N_{1}$ the negative part which is an effective divisor. Then $E_{1} \equiv P_{1}+\left(N_{1}+E_{2}\right)$. Since RHS $-\left(N_{1}+E_{2}\right)$ is movable, the negative part of LHS which is $E_{1}$, satisfies $E_{1} \leq N_{1}+E_{2}$ (cf. [16. ChIII, Proposition 1.14]). Thus $\left(N_{1}+E_{2}-E_{1}\right)$ and also $P_{1}$ are pseudo-effective divisors, but their sum is numerically equivalent to zero. Take general members $H_{i}(1 \leq i \leq n-1)$ in a linear system $|H|$ with $H$ a very ample divisor on $\widetilde{X}$. Then

$$
0=H^{n-1} \cdot\left(P_{1}+N_{1}+E_{2}-E_{1}\right)=H^{n-1} \cdot P_{1}+H^{n-1} \cdot\left(N_{1}+E_{2}-E_{1}\right) .
$$

Hence $H^{n-1} \cdot P_{1}=0=H^{n-1} \cdot\left(N_{1}+E_{2}-E_{1}\right)$. Thus $0=\left(N_{1}+E_{2}-E_{1}\right) \cap\left(H_{1} \cap \cdots \cap H_{n-1}\right)$. Since $N_{1}+E_{2}-E_{1}$ is an effective divisor and the restriction to a subvariety of an ample divisor is still an ample divisor, we get $N_{1}+E_{2}-E_{1}=0$. Thus $N_{1}+E_{2}=E_{1}$. Since $E_{i}$ have no common components, either $E_{2}=0$, or $E_{1}=0$ (and hence $N_{1}=E_{2}=0$ ). So $E_{2}=0$ and hence $K_{\tilde{X}}=\gamma^{*} K_{X}+E_{1}$ with $E_{1} \geq 0$. Therefore, $X$ has at worst canonical singularities by definition. This together with $K_{X} \equiv 0$ imply that $K_{X} \sim_{\mathbb{Q}} 0$ by $[9$, Theorem 8.2].

Lemma 2.5. Let $W$ be an algebraic Lang hyperbolic projective variety, $V$ a projective variety with at worst klt singularities, and $h: V \rightarrow W$ a morphism such that $V \rightarrow h(V)$ is generically finite. Assume that $K_{V}$ is relatively nef over $W$. Then $K_{V}$ is nef.

Proof. Suppose the contrary that $K_{V}$ is not nef and hence there is a $K_{V}$-negative extremal rational curve $C$ by the cone theorem [12, Theorem 3.7]. Since $W$ is hyperbolic and hence contains no rational curve, $C$ must be contracted by $V \rightarrow W$. So $K_{V}$. $C<0$ for a curve $C \subset V$ contracted by $V \rightarrow W$. This contradicts the relative nefness of $K_{V}$ over $W$. Hence $K_{V}$ is nef. This proves the lemma.

Lemma 2.6. Let $W$ be an algebraic Lang hyperbolic projective variety, $X$ a projective variety and $g: X \rightarrow W$ a morphism such that $X \rightarrow g(X)$ is generically finite.

Then there is a birational map $X \rightarrow X_{m}$ over $W$, i.e., there is a (generically finite) morphism $g_{m}: X_{m} \rightarrow W$ such that the natural composition $X \rightarrow X_{m} \stackrel{g_{m}}{\rightarrow} W$ coincides with $g: X \rightarrow W$ (and hence $\left.g_{m}\left(X_{m}\right)=g(X)\right)$ :

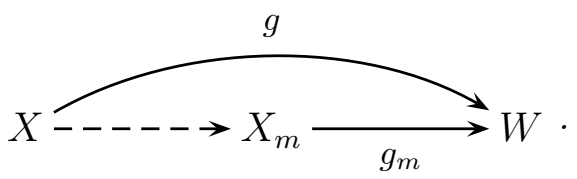


Further, $X_{m}$ has at worst canonical singularities; the canonical divisor $K_{X_{m}}$ is nef; and $K_{X_{m}}$ is also relatively ample over $W$.

Proof. Since $g(X)$ is also hyperbolic, replacing $W$ by $g(X)$, we may assume that $g$ is surjective (and generically finite). Take a projective resolution $X^{\prime} \rightarrow X$. Since the relative dimension of $X^{\prime}$ over $W$ is zero, the canonical divisor $K_{X^{\prime}}$ (and indeed every divisor on $X^{\prime}$ ) is relative big over $W$. The main Theorem 1.2 in [2] says that $X^{\prime}$ has a $\log$ canonical model $X_{m}$ over $W$, so $X_{m}$ has at worst canonical singularities and $K_{X_{m}}$ is relative ample over $W$. This $X_{m}$ is obtained from a log terminal model of $X^{\prime}$ over $W$ followed by a birational morphism over $W$ using the relative-base point freeness result for relative nef and big divisors; see [2, Theorem 1.2, Definition 3.6.7, Theorem 3.9.1]. We note that 2] considers log pairs, while ours is the pure case; so the smoothness of $X^{\prime}$ implies that the log terminal (resp. log canonical) model of $X^{\prime}$ over $W$ has at worst terminal (resp. canonical) singularities. By Lemma 2.5, $K_{X_{m}}$ is nef. This proves the lemma.

Remark 2.7. (1) By the proof, every subvariety $S$ of $X$ (with $g_{\mid S}$ generically finite) or of hyperbolic $W$ has a minimal model $S_{m}$ with only canonical singularities.

(2) Assume $n\left(K_{X_{m}}\right)=\operatorname{dim} X_{m} \geq 1$ and Conjecture 1.3 (2) holds. Then the Kodaira dimension $\kappa\left(X_{m}\right)>0$. By [10, Theorem 7.3], $K_{X_{m}}$ is "good" (or abundant). So it is semi-ample by [10, Theorem 1.1], which has a new proof by Fujino.

(3) Suppose that $Y$ is a normal projective variety birational to the $X$ in Lemma 2.6 and $K_{Y}$ is $\mathbb{Q}$-Cartier. Then $K_{Y}$ is pseudo-effective. Indeed, let $\sigma: Y^{\prime} \rightarrow Y$ be a resolution. Since $g: X \rightarrow W$ is generically finite and $W$ is hyperbolic, $X$ and hence $Y$ and $Y^{\prime}$ are non-uniruled. By [3, Theorem 2.6], $K_{Y^{\prime}}$ is pseudo-effective. Hence $K_{Y}=\sigma_{*} K_{Y^{\prime}}$ is pseudo-effective.

\section{Proof of Theorems}

In this section, we prove results in Introduction, and Theorems 3.1 and 3.2 which imply Theorem 1.5 and Proposition 1.7, respectively. We also prove Corollaries 3.7 and 3.8, all in dimension $\leq 3$, where we do not assume Conjecture 1.1 or 1.3 .

When $\operatorname{dim} X \leq 3$, Case (3) below does not occur.

Theorem 3.1. Let $W$ be an algebraic Lang hyperbolic projective variety, $X$ a projective variety of dimension $n$ and $g: X \rightarrow W$ a morphism such that $X \rightarrow g(X)$ is generically finite. Assume either $n \leq 3$ or Conjecture 1.3(2) (resp. either $n \leq 3$ or Conjecture 1.3(2) without the extra Hyp $(A))$ holds for all varieties birational to $X$. 
Then there is a birational map $X \rightarrow X_{m}$ over $W$, i.e., there is a morphism $g_{m}$ : $X_{m} \rightarrow W$ such that the composition $X \rightarrow X_{m} \stackrel{g_{m}}{\rightarrow} W$ coincides with $g: X \rightarrow W$ (and hence $\left.g_{m}\left(X_{m}\right)=g(X)\right)$ :

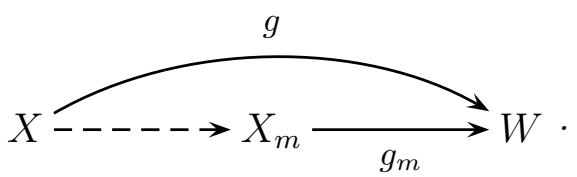

Further, $X_{m}$ is a minimal variety with at worst canonical singularities; $K_{X_{m}}$ is relatively ample over $W$; and one of the following is true.

(1) $K_{X_{m}}$ is ample. Hence both $X_{m}$ and $X$ are of general type.

(2) $X_{m}$ is an absolutely minimal Calabi-Yau variety of dimension $n \geq 3$, and $g_{m}$ : $X_{m} \rightarrow g_{m}\left(X_{m}\right)=g(X) \subseteq W$ is a finite morphism.

(3) There is an almost holomorphic map $\tau: X_{m} \rightarrow Y$ (resp. a holomorphic map $\left.\tau: X_{m} \rightarrow Y\right)$ such that its general fibre $F$ is an absolutely minimal Calabi-Yau variety with $3 \leq \operatorname{dim} F<\operatorname{dim} X_{m}$, and $\left(g_{m}\right)_{\mid F}: F \rightarrow g_{m}(F) \subset g_{m}\left(X_{m}\right)=g(X) \subseteq$ $W$ is a finite morphism. The Kodaira dimension $\kappa(X) \leq \operatorname{dim} Y \leq n-3$.

In Theorem 3.2 below, Conjecture 1.1 or 1.3 is not assumed. a $(W)$ denotes (the Zariskiclosure of $)$ the image $\operatorname{Im}\left(\operatorname{alb}_{W}: W \rightarrow \operatorname{Alb}(W)\right)$ of the albanese map. Since $\operatorname{Alb}(W)$ is generated by a $(W)$, and $\operatorname{dim} \operatorname{Alb}(W)=q(\widetilde{W})=\frac{1}{2} b_{1}(\widetilde{W})$ for any projective resolution $\widetilde{W}$ of $W$, the condition (iii) in Theorem 3.2 is satisfied if $n=4$ and $q(\widetilde{W})>0$.

For related work, the authors of [7] also considered albanese map for smooth $W$ and used classical results of Ueno, [8, Theorem 1], etc., while we use [5], [9], [10].

Theorem 3.2. Let $X$ be an algebraic Lang hyperbolic projective variety of dimension $n$. Assume one of the following conditions holds.

(i) $X$ has maximal albanese dimension, i.e., $\operatorname{dim} \mathrm{a}(X)=\operatorname{dim} X$.

(ii) The Kodaira dimension $\kappa(X) \geq n-3$.

(iii) $\operatorname{dim} \mathrm{a}(X) \geq n-3$ and $\kappa(\mathrm{a}(X)) \geq n-4$.

Then one of the following is true.

(1) There is a birational surjective morphism $g_{m}: X_{m} \rightarrow X$ such that $X_{m}$ has at worst canonical singularities, $K_{X_{m}}$ is ample and hence both $X_{m}$ and $X$ are of general type.

(2) $\kappa(X) \in\{n-3, n-4\}$, and $X$ is covered by subvarieties whose normalizations are absolutely minimal Calabi-Yau varieties of dimension three.

We prove Theorem 1.4. Let $f^{\prime \prime}: X^{\prime \prime} \rightarrow X$ be a dlt blowup with $E_{f^{\prime \prime}}$ the reduced $f^{\prime \prime}$-exceptional divisor (cf. [4, Theorem 10.4]). Namely, $X^{\prime \prime}$ is $\mathbb{Q}$-factorial, $\left(X^{\prime \prime}, E_{f^{\prime \prime}}\right)$ is dlt 
(and hence $X^{\prime \prime}$ is klt) and

$$
\left(f^{\prime \prime}\right)^{*} K_{X}=K_{X^{\prime \prime}}+E^{\prime \prime}
$$

where $E^{\prime \prime}$ is $f^{\prime \prime}$-exceptional and satisfies $E^{\prime \prime} \geq E_{f^{\prime \prime}}$.

Since $f^{\prime \prime}$ is birational, $K_{X^{\prime \prime}}$ is relative big over $X$. By [2, Theorem 1.2, Definition 3.6.7], there is a birational map $\sigma: X^{\prime \prime} \rightarrow X^{\prime}$ over $X$, such that $\sigma^{-1}$ does not contract any divisor, $X^{\prime}$, like $X^{\prime \prime}$, has only $\mathbb{Q}$-factorial klt singularities and $K_{X^{\prime}}$ is relatively nef over $X$ via a birational morphism $f^{\prime}: X^{\prime} \rightarrow X$. Pushing forward the equality (1) above by $\sigma_{*}$, we get

$$
\left(f^{\prime}\right)^{*} K_{X}=K_{X^{\prime}}+E^{\prime}
$$

where $E^{\prime}:=\sigma_{*} E^{\prime \prime} \geq \sigma_{*} E_{f^{\prime \prime}}=E_{f^{\prime}}$ and $E_{f^{\prime}}$ is the reduced $f^{\prime}$-exceptional divisor. Since $K_{X^{\prime}}$ is relatively $f^{\prime}$-nef over $X$, our $K_{X^{\prime}}$ is nef by Lemma 2.5.

Claim 3.3. $K_{X^{\prime}}$ is nef.

We continue the proof of Theorem 1.4. Let $\tau: X^{\prime} \rightarrow Y$ be a nef reduction of the nef divisor $K_{X^{\prime}}$, and $n\left(K_{X^{\prime}}\right):=\operatorname{dim} Y$ the nef dimension of $K_{X^{\prime}}$; let $F$ be a general (compact) fibre of $\tau$; then $K_{F}=\left(K_{X^{\prime}}\right)_{\mid F}$ is numerically trivial (cf. Theorem 1.2).

Lemma 3.4. Assume the hypotheses of Theorem 1.4. For the $X^{\prime}$ and $\tau: X^{\prime} \rightarrow Y$ defined above, it is impossible that $\operatorname{dim} Y=0$.

Proof. Consider the case $\operatorname{dim} Y=0$. Then $K_{X^{\prime}} \equiv 0$ (numerically zero). Since $X$ is hyperbolic, $X$ and hence $X^{\prime}$ are non-uniruled. By Lemma 2.4, $X^{\prime}$ has at worst canonical singularities, and $K_{X^{\prime}} \sim_{\mathbb{Q}} 0$; the same hold for $X$, noting that $K_{X}=f_{*}^{\prime} K_{X^{\prime}} \sim_{\mathbb{Q}} 0$ (cf. [11, Corollary 1.5]).

We claim that $X$ is a Calabi-Yau variety. We only need to show that the irregularity $q(X)=0$. Suppose the contrary that $q(X)>0$. Then, by Lemma $2.3, X$ is covered by images of abelian varieties of dimension equal to $q(X)$. This contradicts the hyperbolicity of $X$. Therefore, $q(X)=0$. Hence $X$ is a Calabi-Yau variety. This contradicts the hyperbolicity of $X$, Remark 1.8 and the assumed Conjecture 1.1. This proves Lemma 3.4 .

Lemma 3.5. Assume the hypotheses of Theorem 1.4. For the $X^{\prime}$ and $\tau: X^{\prime} \rightarrow Y$ defined preceding Lemma 3.4, it is impossible that $1 \leq \operatorname{dim} Y<\operatorname{dim} X^{\prime}$.

Proof. Consider the case $1 \leq \operatorname{dim} Y<\operatorname{dim} X^{\prime}$. A general fibre $F$ of $\tau: X^{\prime} \rightarrow Y$ satisfies $1 \leq \operatorname{dim} F=\operatorname{dim} X-\operatorname{dim} Y<\operatorname{dim} X$. Also $K_{F} \equiv 0$. Since $X$ and hence the general fibre $F$ of $\tau: X^{\prime} \rightarrow Y$ are not covered by rational curves by the hyperbolicity of $X, F$ is not uniruled. By Lemma 2.4, $F$ has at worst canonical singularities and $K_{F} \sim_{\mathbb{Q}} 0$. 
Factor the birational map $X^{\prime} \supset F \rightarrow f^{\prime}(F) \subset X$ as $F \rightarrow F^{n} \rightarrow f^{\prime}(F)$, where $F \rightarrow F^{n}$ is a birational morphism and $F^{n} \rightarrow f^{\prime}(F)$ is the normalization. By [11, Corollary 1.5], $F^{n}$ has only canonical singularities and $K_{F^{n}} \sim_{\mathbb{Q}} 0$.

If $q\left(F^{n}\right)>0$, by Lemma 2.3, $F^{n}$ and hence $f^{\prime}(F)$ and $X$ are covered by images of abelian varieties of dimension equal to $q\left(F^{n}\right)$, contradicting the hyperbolicity of $X$. Thus $q\left(F^{n}\right)=0$, so $F^{n}$ is a Calabi-Yau variety. By the assumed Conjecture 1.1 and Remark 1.8, there is a non-constant holomorphic map $V \rightarrow F^{n}$ from an abelian variety $V$, which, combined with the (birational and) finite map $F^{n} \rightarrow f^{\prime}(F)$, produces a non-constant holomorphic map $V \rightarrow X$, contradicting the hyperbolicity of $X$. This proves Lemma 3.5 .

By the two lemmas above, we are left with the case $\operatorname{dim} Y=\operatorname{dim} X^{\prime}$. Namely, the nef dimension $n\left(K_{X^{\prime}}\right)=\operatorname{dim} X^{\prime}$. By the assumed abundance Conjecture $1.3(1), K_{X^{\prime}}$ is semi-ample. Hence $\Phi_{\left|s K_{X^{\prime}}\right|}$, for some $s>0$, is a morphism onto a normal variety $X_{c}$, with connected fibres, and there is an ample $\mathbb{Q}$-divisor $H_{c}$ on $X_{c}$ such that $K_{X^{\prime}} \sim_{\mathbb{Q}}$ $\Phi_{\left|s K_{X^{\prime}}\right|}^{*} H_{c}$. Clearly, this map which is now holomorphic, is (up to birational equivalence) a nef reduction of $K_{X^{\prime}}$ and also denoted as $\tau: X^{\prime} \rightarrow X_{c}$. In other words, $Y=X_{c}, K_{X^{\prime}}$ is big (and nef), and $\tau$ is birational. Pushing forward the equality $K_{X^{\prime}} \sim_{\mathbb{Q}} \tau^{*} H_{c}$ by $\tau_{*}$, we get $K_{X_{c}} \sim_{\mathbb{Q}} H_{c}$ and hence $K_{X^{\prime}} \sim_{\mathbb{Q}} \tau^{*} K_{X_{c}}$ (so that $\tau$ is a crepant birational morphism) with $K_{X_{c}}$ an ample $\mathbb{Q}$-divisor. Since $X^{\prime}$ is klt and $\tau$ is crepant, $X$ is also klt. By [6, Corollary 1.5], every fibre of $\tau: X^{\prime} \rightarrow X_{c}$ is rationally chain connected and hence is contracted to a point by the birational morphism $f^{\prime}: X^{\prime} \rightarrow X$ due to the hyperbolicity of $X$ and the absence of rational curves on $X$. Thus $f^{\prime}$ factors through $\tau$, i.e., there is a birational morphism $f_{c}: X_{c} \rightarrow X$ such that $f^{\prime}=f_{c} \circ \tau$ (cf. [8, Proof of Lemma 14]). In summary, we have the following commutative diagram:

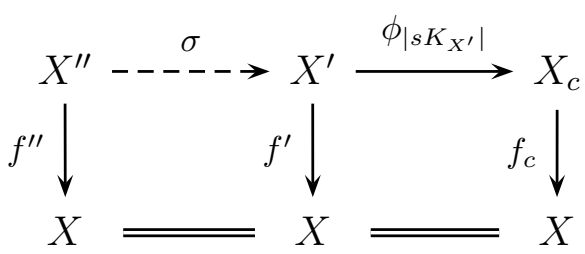

Pushing forward the equality (2) above by $\tau_{*}$, we get

$$
f_{c}^{*} K_{X}=K_{X_{c}}+E_{c}
$$

Here $E_{c}:=\tau_{*} E^{\prime} \geq \tau_{*} E_{f^{\prime}}=E_{f_{c}}$ and $E_{f_{c}}$ is the reduced $f_{c^{-}}$exceptional divisor. So the image $f_{c}\left(E_{c}\right)$ is contained in $\operatorname{Nklt}(X)$, the non-klt locus of $X$, which is a Zariski-closed subset of $X$ consisting of exactly the non-klt points of $X$.

This proves Theorem 1.4; see Remark 1.8 (1) for the final part. 
If we do not assume Conjecture 1.1] in Theorem 1.4, the argument above actually shows:

Remark 3.6. Let $X$ be a $\mathbb{Q}$-Gorenstein normal projective variety of dimension $n$ which is algebraic Lang hyperbolic. Assume either $n \leq 3$ or Conjecture 1.3(1) holds for all varieties of dimension $\leq n$. Then either $K_{X}$ is ample at smooth points and klt points of $X$ as detailed in Theorem 1.4; ; or $X$ is a Calabi-Yau variety of dimension $n \geq 3$; or $X$ is covered by subvarieties $\left\{F_{t}^{\prime}\right\}$ whose normalizations are Calabi-Yau varieties of the same dimension $k$ with $3 \leq k<n$.

Since Conjecture 1.1 is true for surfaces, and the abundance Conjecture 1.3 is known in dimension $\leq 3$, we can and will soon prove the following consequences of Theorem 1.4 .

Corollary 3.7. Let $X$ be a $\mathbb{Q}$-Gorenstein normal projective surface which is algebraic Lang hyperbolic. Then $X$ is of general type and the canonical divisor $K_{X}$ is ample.

We can not remove the second alternative below even when $X$ is smooth, because, for instance, we do not know, at the moment, the non-hyperbolicity of a general smooth Calabi-Yau $n$-fold and a Hyperkähler $n$-fold when $n>2$.

Corollary 3.8. Let $X$ be a $\mathbb{Q}$-Gorenstein normal projective threefold which is algebraic Lang hyperbolic. Then either the canonical divisor $K_{X}$ is ample at the smooth points and klt points of $X$; or $X$ is a Calabi-Yau variety.

\subsection{Proof of Corollaries $\mathbf{3 . 7}$ and $\mathbf{3 . 8}$}

We use the fact that Conjecture 1.1 holds in dimension $\leq 2$, and both Conjectures 1.3 (1) and (2) (even without the extra $\operatorname{Hyp}(\mathrm{A})$ ) hold in dimension $\leq 3$ (cf. Proposition 2.2).

Corollary 3.7 is a consequence of Theorem 1.4 and the observation: if $f_{c}: X_{c} \rightarrow X$ is a birational morphism between $\mathbb{Q}$-Gorenstein normal projective surfaces and $K_{X_{c}}$ is ample, then $K_{X}=\left(f_{c}\right)_{*} K_{X_{c}}$ is also ample, by using Nakai-Moishezon ampleness criterion and the projection formula.

For Corollary 3.8, we follow the argument for the proof of Theorem 1.4, Thus we have to consider Cases (I) $\operatorname{dim} Y=0$, (II) $0<\operatorname{dim} Y<\operatorname{dim} X$ and (III) $\operatorname{dim} Y=\operatorname{dim} X$. In Case (I), $X$ has been proven to be a Calabi-Yau variety of dimension three; for this purpose, Conjecture 1.1 was not used. In Case (II), a contradiction has been deduced utilizing Conjecture 1.1 in dimension $\leq 2$. In Case (III), using the proven abundance conjecture in dimension 3 , we get the first possibility in the conclusion part of Corollary 3.8. This proves Corollary 3.8 .

\subsection{Proof of Theorems 1.5 and 3.1}


If we let $g: X \rightarrow W$ in Theorem 3.1 be the identity map $\operatorname{id}_{X}: X \rightarrow X$, we get Theorem 1.5, we also use the observation that a birational finite morphism from a normal variety like $X_{m}$ or $F$ in Theorem 3.1 is just the normalization map. Thus we have only to (and are going to) prove Theorem 3.1 .

We may assume Conjecture 1.3 (2) (the case without the extra $\operatorname{Hyp}(\mathrm{A})$ is similar and indeed easier); for varieties of dimension $\leq 3$, this assumption is automatically satisfied by Proposition 2.2.

Theorem 3.1 is clearly true when $\operatorname{dim} X=1$. So we may assume that $n=\operatorname{dim} X \geq 2$. We apply Lemma 2.6 and let the birational map $X \rightarrow X_{m}$ over $W$ and $g_{m}: X_{m} \rightarrow W$ be as there, where $X_{m}$ has only canonical singularities and $K_{X_{m}}$ is relatively ample over $W$ and is also nef. Since $g: X \rightarrow W$ is generically finite, so is $g_{m}: X_{m} \rightarrow W$.

Let $\tau: X_{m} \rightarrow Y$ be a nef reduction of the nef divisor $K_{X_{m}}$. For our $\tau: X_{m} \rightarrow Y$ and $g_{m}: X_{m} \rightarrow W$ here (with $W$ algebraic Lang hyperbolic) we closely follow the argument of Theorem 1.4 for $\tau: X^{\prime} \rightarrow Y$ and $f^{\prime}: X^{\prime} \rightarrow X$ there (with $X$ algebraic Lang hyperbolic), but we do not assume Conjecture 1.1 here.

Suppose that $\operatorname{dim} Y=0$, i.e., $n\left(K_{X_{m}}\right)=0$, or $K_{X_{m}} \equiv 0$. Now Lemma 3.4 is applicable under the current weaker assumption. Precisely, due to the lack of the assumption of Conjecture 1.1 here, instead of the contradiction there, we have that $X_{m}$ is a Calabi-Yau variety. The relative ampleness of $K_{X_{m}}$ over $W$ implies that $g_{m}: X_{m} \rightarrow g_{m}\left(X_{m}\right)=$ $g(X) \subseteq W$ is a finite morphism. This and the hyperbolicity of $W$ imply that $X_{m}$ is hyperbolic. Further, $X_{m}$ is absolutely minimal (cf. Remark 1.8). By Proposition 2.2, $\operatorname{dim} X_{m} \geq 3$. So Case 3.1 (2) occurs.

Suppose that $1 \leq n\left(K_{X_{m}}\right)<n$. Denote by $F$ a general fibre of $\tau: X_{m} \rightarrow Y$. For this case, Lemma 3.5 is applicable even under the current weaker assumption. So $F$ is a Calabi-Yau variety. The map $\left(g_{m}\right)_{\mid F}: F \rightarrow g_{m}(F) \subset g_{m}\left(X_{m}\right)=g(X) \subseteq W$ is a finite morphism, otherwise, a curve $C$ in $F$ is $g_{m}$-exceptional and hence $K_{X_{m}} . C>0$, by the relative ampleness of $K_{X_{m}}$ over $W$, contradicting the numerical triviality of $\left(K_{X_{m}}\right)_{\mid F}$ entailing $K_{X_{m}} . C=0$. This and the hyperbolicity of $W$ imply that $F$ is hyperbolic. Further, $F$ is absolutely minimal (cf. Remark 1.8). By Proposition [2.2, $\operatorname{dim} F \geq 3$. So Case 3.1 (3) occurs. Indeed, for the final part, the well known Iitaka addition for Kodaira dimension implies that $\kappa(X) \leq \kappa(F)+\operatorname{dim} Y=\operatorname{dim} Y=n-\operatorname{dim} F \leq n-3$.

Suppose that $n\left(K_{X_{m}}\right)=n$. Then $K_{X_{m}}$ is semi-ample by Conjecture1.3 (2) (cf. Remark 2.7). Hence $K_{X_{m}}$ is nef and big, and there is a birational morphism $\gamma: X_{m} \rightarrow Z$ onto a normal variety $Z$ such that $K_{X_{m}} \sim_{\mathbb{Q}} \gamma^{*} H$ for an ample $\mathbb{Q}$-divisor $H$ on $Z$. Hence both $X_{m}$ and $X$ are of general type. Since $\gamma$ is birational, the projection formula implies that $K_{Z}=\gamma_{*} K_{X_{m}} \sim_{\mathbb{Q}} H$. Hence $K_{Z}$ is ample and $K_{X_{m}} \sim_{\mathbb{Q}} \gamma^{*} K_{Z}$. Thus $Z$, like $X_{m}$, has only canonical singularities. If $\gamma$ is not an isomorphism, then it has a positive-dimensional 
fibre. By [6, Corollary 1.5], every fibre of $\gamma$ is rationally chain connected. So we may assume that $\gamma$ contracts a rational curve $C$ on $X_{m}$ to a point on $Z$. Since $W$ is hyperbolic, $g_{m}: X_{m} \rightarrow X \subseteq W$ contracts the rational curve $C$ on $X_{m}$ to a point on $W$. This and $C . K_{X_{m}}=C \cdot \gamma^{*} K_{Z}=\gamma_{*} C . K_{Z}=0$ contradict the relative ampleness of $K_{X_{m}}$ over $W$. So $\gamma: X_{m} \rightarrow Z$ is an isomorphism. Hence $K_{X_{m}}$ is ample. Thus Case 3.1 (1) occurs. This proves Theorem 3.1 .

\subsection{Proof of Corollary $\mathbf{1 . 6}$}

For the assertion (1), we apply Theorem 3.1 to the inclusion map $g: X \hookrightarrow W$ for a projective subvariety $X$ of $W$. By Theorem 3.1, either Case 3.1 (1) occurs and hence $X$ is of general type, or Case 3.1 (2) or (3) occurs. We use the the notation there: birational morphism $g_{m}: X_{m} \rightarrow X$, etc. If Case 3.1 (2) (resp. (3)) occurs, by the assumed Conjecture 1.1 and Remark 1.8, there is a non-constant holomorphic map from an abelian variety $V$ to $X_{m}$ (resp. to $F$ ), thus, combined with the (birational and) finite morphism

$$
g_{m}: X_{m} \rightarrow g_{m}\left(X_{m}\right)=g(X)=X \subseteq W
$$

(resp. $\left(g_{m}\right)_{\mid F}: F \rightarrow g_{m}(F) \subset g_{m}\left(X_{m}\right)=g(X)=X \subseteq W$ ), this map gives a non-constant holomorphic map from $V$ to $W$, contradicting the hyperbolicity of $W$. This proves the assertion (1).

For the assertion (2), the case $\operatorname{dim} W=1$ is clear. We may assume that $\operatorname{dim} W \geq 2$. We apply Theorem 3.1 and let $g: X \rightarrow W$ be the identity map id $X: X \rightarrow X$, with $W=X$. Hence there is a birational morphism $g_{m}: X_{m} \rightarrow X$ such that 3.1 (1), (2) or (3) occurs. We use the following known fact (cf. [9, Lemma 8.1], or Lemma 2.3):

Fact 3.12. If a projective variety $V$ has only canonical (or more generally rational) singularities, then the albanese map $\mathrm{alb}_{V}: V \rightarrow \operatorname{Alb}(V)$ is a well defined morphism and $\operatorname{dim} \operatorname{Alb}(V)=q(V)=h^{1}\left(V, \mathcal{O}_{V}\right)$.

In Case 3.1 (1), $X$ is of general type.

In Case 3.1 (2), the irregularity $q\left(X_{m}\right)=0$, and hence $\operatorname{Alb}(X)=\operatorname{Alb}\left(X_{m}\right)$ is a point. Thus $X$ itself is the fibre of $\operatorname{alb}_{X}$. Hence $\operatorname{dim} X \leq 2$, by the assumption. Thus $\operatorname{dim} X=2$, by the extra assumption that $X=W$ has dimension at least two. By Proposition 2.2, either $X$ has infinitely many rational or elliptic curves, or it is birational to an abelian surface (and hence $q\left(X_{m}\right)=2$ ), or it is of general type. Since $X=W$ is hyperbolic and $q\left(X_{m}\right)=0, X=W$ is of general type.

In Case 3.1 (3), by the above fact, $\operatorname{alb}_{X_{m}}: X_{m} \rightarrow \operatorname{Alb}\left(X_{m}\right)=\operatorname{Alb}(X)$ is a well defined morphism. Since the general fibre $F$ of the nef reduction map $\tau: X_{m} \rightarrow Y$ which is almost holomorphic, is a Calabi-Yau variety, we have $q(F)=0$, so $\operatorname{Alb}(F)$ is a point. 
By the universality of the albanese map, the composition $F \hookrightarrow X_{m} \rightarrow \operatorname{Alb}\left(X_{m}\right)$ factors through $F \rightarrow \operatorname{Alb}(F)$. So $\operatorname{alb}_{X_{m}}: X_{m} \rightarrow \operatorname{Alb}\left(X_{m}\right)$ maps $F$ to a point. Hence $g_{m}(F) \subset X$ is contained in a general fibre $G$ of $\operatorname{alb}_{X}: X \stackrel{g_{m}^{-1}}{\rightarrow} X_{m} \rightarrow \operatorname{Alb}\left(X_{m}\right)=\operatorname{Alb}(X)$. Indeed, alb $_{X}$ factors as

$$
X \stackrel{g_{m}^{-1}}{\rightarrow-\rightarrow} X_{m} \stackrel{\tau}{-\rightarrow} Y \stackrel{\eta}{\rightarrow} Y \operatorname{Alb}\left(X_{m}\right)=\operatorname{Alb}(X)
$$

for some rational map $\eta$, by applying [8, Lemma 14] to the domain of the almost holomorphic map $\tau$. Now $\operatorname{dim} G \geq \operatorname{dim} g_{m}(F)=\operatorname{dim} F \geq 3$, with the last inequality shown in Theorem 3.1. This contradicts the assumption. This proves Corollary 1.6.

\subsection{Proof of Proposition 1.7 and Theorem 3.2}

We prove Theorem 3.2. We first assume that $X$ has maximal albanese dimension. Since abundance conjecture holds for varieties with maximal albanese dimension (cf. Proposition 2.2), we can apply Theorem 1.5 to our $X$. So Case 1.5 (1), (2) or (3) occurs. We use the notation $g_{m}: X_{m} \rightarrow X$ there, where $K_{X_{m}}$ is relatively ample over $X$, and is also nef. By [5, Theorem 3.6], $K_{X_{m}}$ is semi-ample. So there exist a holomorphic map $\tau:=\Phi_{\left|s K_{X_{m} \mid}\right|}: X_{m} \rightarrow Y$ onto a normal variety $Y$, for some $s>0$, and an ample divisor $H$ on $Y$ such that $K_{X_{m}} \sim_{\mathbb{Q}} \tau^{*} H$. We can take this $\tau$ as a nef reduction of $K_{X_{m}}$ in Theorem 1.5. Let $F$ be a general fibre of $\tau$.

If Case 1.5 (1) occurs, we are in Case 3.2 (1).

Consider Case $1.5(3)$. Then $\left(g_{m}\right)_{\mid F}: F \rightarrow g_{m}(F)$ is the normalization map, and $F \subseteq X_{m}$ is a Calabi-Yau variety of dimension $\geq 3$. Since $X$ and hence $X_{m}$ have maximal albanese dimension, i.e., $\operatorname{dim} \mathrm{a}\left(X_{m}\right)=\operatorname{dim} X_{m}$, we may assume that the restriction $\left(\operatorname{alb}_{X_{m}}\right)_{\mid F}$ to a general fibre $F$ of $\tau$, is a generically finite morphism onto the image $F_{a} \subseteq \operatorname{Alb}(X)$. It is known that $F_{a}$, being a subvariety of an abelian variety, satisfies $\kappa\left(F_{a}\right) \geq 0$ with equality holding only when $F_{a}$ is the translation of a subtorus. Now $0=\kappa(F) \geq \kappa\left(F_{a}\right) \geq 0$. Thus $\kappa\left(F_{a}\right)=0$ and hence $F_{a}$ is an abelian variety. Therefore, $0=q(F) \geq q\left(F_{a}\right)=\operatorname{dim} F_{a}=\operatorname{dim} F \geq 3$. This is a contradiction.

If Case 1.5 (2) occurs, we get a similar contradiction by the arguments above, with $F=X_{m}$.

Next we assume that $\kappa(X) \geq n-3$. Let $I_{X}: X \rightarrow Y$ be the Iitaka fibration with $F$ a very general fibre. So $\kappa(F)=0$ and $\operatorname{dim} F=\operatorname{dim} X-\kappa(X) \leq 3$ by the assumption. Since $F$ is a subvariety of the hyperbolic variety $X$, it is also hyperbolic.

If $\operatorname{dim} F=0$, then $X$ is of general type. Since abundance conjecture holds for varieties of general type (cf. Proposition 2.2), we can apply Theorem 1.5 to our $X$, and only Case 1.5 (1) there occurs. This fits Case 3.2 (1). 
Consider the case $\operatorname{dim} F \in\{1,2,3\}$. Applying Theorem 1.5 to the hyperbolic $F$ and noting that $\kappa(F)=0$ and $1 \leq \operatorname{dim} F \leq 3$, only Case 1.5 (2) there occurs for $F$ : the normalization of $F$ is an absolutely minimal Calabi-Yau variety of dimension three, so $\kappa(X)=n-\operatorname{dim} F=n-3$; also these $F$ cover $X$. This fits Case 3.2 (2).

Finally, we assume that $\operatorname{dim} \mathrm{a}(X) \geq n-3$ and $\kappa(\mathrm{a}(X)) \geq n-4$. By the results obtained so far, we may add the extra assumptions: $n>\operatorname{dim} \mathrm{a}(X)$ and $\kappa(X) \leq n-4$. Let $G$ be an irreducible component of a general fibre of the albanese map $\operatorname{alb}_{X}: X-\rightarrow$ $\mathrm{a}(X) \subseteq \operatorname{Alb}(X)$. Then $1 \leq g:=\operatorname{dim} G=\operatorname{dim} X-\operatorname{dim} \mathrm{a}(X) \leq 3$. The hyperbolicity of $X$ implies that $X$ and hence $G$ are not uniruled. So $G$ has a good minimal model in the sense of Kawamata [10], by the abundance theorem in dimension $\leq 3$ (cf. [12, §3.13] or Proposition 2.2). In particular, $\kappa(G) \geq 0$. By Iitaka's $C_{n, n-g}$ proved in [9, Corollary 1.2], $\kappa(X) \geq \kappa(G)+\kappa(\mathrm{a}(X)) \geq 0+0$. By the assumptions,

$$
n-4 \geq \kappa(X) \geq \kappa(G)+\kappa(\mathrm{a}(X)) \geq \kappa(G)+n-4 \geq n-4 .
$$

Thus the inequalities above all become equalities. In particular, $\kappa(G)=0$ and $\kappa(X)=$ $n-4$. Since $\kappa(X) \geq 0$, we have $n \geq 4$. As in the previous paragraph, applying Theorem 1.5 to $G$, the normalization of $G$ is an absolutely minimal Calabi-Yau variety of dimension three; also these $G$ cover $X$. This fits Case 3.2 (2).

This proves Theorem 3.2 .

Now we prove Proposition 1.7. Set $n:=\operatorname{dim} X$. Each of the three conditions in Proposition 1.7 implies one of the first two conditions in Theorem 3.2. Hence we can apply Theorem 3.2. We may assume that Case 3.2 (2) occurs. Thus $X$ is covered by subvarieties $F_{t}$ whose normalizations $F_{t}^{n}$ are absolutely minimal Calabi-Yau varieties of dimension three. The algebraic Lang hyperbolicity of $X$ implies the same for $F_{t}$ and also $F_{t}^{n}$. This contradicts the assumed Conjecture 1.1 in dimension three (cf. Remark 1.8).

This proves Proposition 1.7 .

\section{REFERENCES}

[1] T. Bauer, F. Campana, T. Eckl, S. Kebekus, T. Peternell, S. Rams, T. Szemberg and L. Wotzlaw, A reduction map for nef line bundles, Complex geometry (Göttingen, 2000), 27 - 36, Springer, 2002.

[2] C. Birkar, P. Cascini, C. D. Hacon and J. McKernan, Existence of minimal models for varieties of log general type, J. Amer. Math. Soc. 23 (2010) $405-468$.

[3] S. Boucksom, J. -P. Demailly, M. Paun and T. Peternell, The pseudo-effective cone of a compact Kähler manifold and varieties of negative Kodaira dimension, J. Algebraic Geom. 22 (2013), no. 2, 201-248.

[4] O. Fujino, Fundamental theorems for the log minimal model program, Publ. Res. Inst. Math. Sci. 47 (2011), no. $3,727-789$. 
[5] O. Fujino, On maximal Albanese dimensional varieties, Proc. Japan Acad. Ser. A Math. Sci. 89 (2013), no. 8, $92-95$.

[6] C. D. Hacon and J. McKernan, On Shokurov's rational connectedness conjecture, Duke Math. J. 138 (2007), no. 1, $119-136$.

[7] G. Heier, Steven S. Y. Lu and B. Wong, On the canonical line bundle and negative holomorphic sectional curvature, Math. Res. Lett. 17 (2010), no. 6, $1101-1110$.

[8] Y. Kawamata, Characterization of abelian varieties, Compositio Math. 43 (1981), no. 2, 253 - 276.

[9] Y. Kawamata, Minimal models and the Kodaira dimension of algebraic fiber spaces, J. Reine Angew. Math. 363 (1985), 1-46.

[10] Y. Kawamata, Pluricanonical systems on minimal algebraic varieties, Invent. Math. 79 (1985), no. $3,567-588$.

[11] Y. Kawamata, Crepant blowing-ups of 3-dimensional canonical singularities and its application to degeneration of surfaces, Ann. of Math. 127 (1988) 93 - 163.

[12] J. Kollár and S. Mori, Birational geometry of algebraic varieties, Cambridge Tracts in Math. 134, Cambridge Univ. Press, 1998.

[13] S. Lang, Hyperbolic and Diophantine analysis, Bull. Amer. Math. Soc. (N.S.) 14 (1986), 159-205.

[14] Steven S. Y. Lu and D. -Q. Zhang, Positivity criteria for log canonical divisors and hyperbolicity, arXiv:1207.7346

[15] S. Mori and S. Mukai, The uniruledness of the moduli space of curves of genus 11, Algebraic geometry (Tokyo/Kyoto, 1982), 334 - 353, Lecture Notes in Math., 1016, Springer, Berlin, 1983.

[16] N. Nakayama, Zariski-decomposition and abundance, MSJ Memoirs Vol.14, Math.Soc. Japan, 2004.

[17] T. Peternell, Calabi-Yau manifolds and a conjecture of Kobayashi, Math. Z. 207 (1991), no. 2, $305-318$.

Department of Mathematics

National University of Singapore, 10 Lower Kent Ridge Road, Singapore 119076

E-mail address: hf@nus.edu.sg

Department of Mathematics

National University of Singapore, 10 Lower Kent Ridge Road, Singapore 119076

E-mail address: ms@nus.edu.sg

Department of Mathematics

National University of Singapore, 10 Lower Kent Ridge Road, Singapore 119076

E-mail address: matzdq@nus.edu.sg 